SISSA/70/2002/EP

SLAC-PUB-9593

hep-ph/0211341

October 28, 2018

\title{
On the Effective Mass of the Electron Neutrino in Beta Decay *
}

\author{
Y. Farzan ${ }^{1,2}$ and A. Yu. Smirnov ${ }^{3,4}$ \\ ${ }^{1}$ Scuola Internazionale Superiore di Studi Avanzati, SISSA, I-34014, Trieste, Italy \\ 2 Stanford Linear Accelerator Center, Stanford University, 2575 Sand Hill Road, Menlo \\ Park, California 94025 \\ ${ }^{3}$ The Abdus Salam International Centre for Theoretical Physics, I-34100 Trieste, Italy \\ ${ }^{4}$ Institute for Nuclear Research, RAS, Moscow, Russia
}

\begin{abstract}
In the presence of mixing between massive neutrino states, the distortion of the electron spectrum in beta decay is, in general, a function of several masses and mixing angles. For $3 \nu$-schemes which describe the solar and atmospheric neutrino data, this distortion can be described by a single effective mass, under certain conditions. In the literature, two different definitions for the effective mass have been suggested. We show that for quasi-degenerate mass schemes (with an overall mass scale $m$ and splitting $\left.\Delta m^{2}\right)$ the two definitions coincide up to $\left(\Delta m^{2}\right)^{2} / m^{4}$ corrections. We consider the impact of different effective masses on the integral energy spectrum. We show that the spectrum with a single mass can be used also to fit the data in the case of $4 \nu$-schemes motivated, in particular, by the LSND results. In this case the accuracy of the mass determination turns out to be better than $(10-15) \%$.
\end{abstract}

${ }^{*}$ Work supported, in part, by U. S. Department of Energy, under contract DE-AC03-76SF00515. 


\section{Introduction}

Determination of the absolute scale of neutrino masses is one of the most important and, at the same time, challenging problems in neutrino physics. Currently, the study of the electron energy spectrum near the end point of the Tritium beta decay, ${ }_{1}^{3} \mathrm{H} \rightarrow{ }_{2}^{3} \mathrm{He}+e^{-}+\bar{\nu}_{e}$, is the most sensitive direct method of determining the scale of masses. It is well-known that in the absence of mixing, the energy spectrum of the emitted $e^{-}$is described by

$$
\frac{d N}{d E}=R(E)\left(E_{0}-E\right)\left[\left(E-E_{0}\right)^{2}-m_{\nu}^{2}\right]^{\frac{1}{2}} \Theta\left(E_{0}-E-m_{\nu}\right)
$$

(see e.g. [1]), where $E$ is the energy of the electron, $E_{0}$ is the total decay energy and $R(E)$ is given by

$$
R(E)=G_{F}^{2} \frac{m_{e}^{5}}{2 \pi^{3}} \cos ^{2} \theta_{C}|M|^{2} F(Z, E) p E .
$$

Here $G_{F}$ is the Fermi constant, $p$ is the momentum of the electron, $\theta_{C}$ is the Cabibbo angle and $M$ is the nuclear matrix element. $F(Z, E)$ is a smooth function of energy which describes the interaction of the produced electron in the final state. Both $M$ and $F(Z, E)$ are independent of $m_{\nu}$, and therefore the dependence of the spectrum on $m_{\nu}$ follows only from the phase space factors.

The analysis of the present data $[2,3]$ in terms of Eq. (1) leads to the following bound:

$$
m_{\nu}<2.2 \mathrm{eV}
$$

The forthcoming beta decay experiment, KATRIN [4], with energy resolution $\Delta E \sim 1 \mathrm{eV}$ will be sensitive to neutrino masses down to

$$
m_{\nu} \sim 0.3 \mathrm{eV}
$$

The atmospheric and solar neutrino data provide strong evidence for neutrino mixing. In the presence of mixing, the electron neutrino is a combination of the mass eigenstates $\nu_{i}$ with masses $m_{i}: \nu_{e}=\sum_{i} U_{e i} \nu_{i}$. In this case, instead of Eq. (1), the expression for the spectrum is given by

$$
\frac{d N_{0}}{d E}=R(E) \sum_{i}\left|U_{e i}\right|^{2}\left(E_{0}-E\right)\left[\left(E_{0}-E\right)^{2}-m_{i}{ }^{2}\right]^{\frac{1}{2}} \Theta\left(E_{0}-E-m_{i}\right)
$$

where the step function, $\Theta\left(E_{0}-E-m_{i}\right)$, reflects the fact that a neutrino can be produced only if the available energy is larger than its mass [5]. According to Eq. (4), in general, several mass and mixing parameters should be used to perform a fit of the experimental data. 
However, in the realistic situation a few parameters (possibly even one) is enough. In fact, the number and type of parameters depend on: (i) the type of the neutrino mass spectrum; (ii) the part of the spectrum being measured or the width of the energy interval under consideration:

$$
\delta \equiv E-E_{0}
$$

(iii) the statistics of the experiment; (iv) the energy resolution of the detector, $\Delta E$. For the KATRIN experiment, if there are only three neutrinos and at least one of their masses is in the sensitivity range of KATRIN (see Eq. (3)), the situation will be simpler. In this case the spectrum should be strongly degenerate, i.e., the mass splittings are too small to be resolved $\left(\Delta m_{i j} \ll \Delta E \sim 1 \mathrm{eV}<\delta\right)$.

The mass splitting is given by

$$
\Delta m_{i j} \equiv m_{i}-m_{j}=\frac{m_{i}^{2}-m_{j}^{2}}{m_{i}+m_{j}} \leq \sqrt{\Delta m_{i j}^{2}} \leq 0.05 \mathrm{eV},
$$

where the inequalities hold for any type of spectrum. The bound $0.05 \mathrm{eV}$ corresponds to the neutrino mass splitting $\Delta m_{i j}^{2}=\Delta m_{a t m}^{2}=3 \times 10^{-3} \mathrm{eV}^{2}$. For the range of sensitivity of KATRIN, $m_{\nu} \sim 0.3-2 \mathrm{eV}$, the mass splitting is even smaller:

$$
\Delta m<5 \times 10^{-3} \mathrm{eV}
$$

and correspondingly,

$$
\frac{\Delta m}{m}<1.6 \times 10^{-2}
$$

Since the energy resolution of the forthcoming experiment, $\Delta E$, will be much larger than $\sqrt{\Delta m_{\text {atm }}^{2}} \sim 0.05 \mathrm{eV}$, the experimental data will not be able to resolve different "kinks" associated with the different mass states and, in spite of the presence of mixing, the spectrum can effectively be described by Eq. (1) with a single effective mass $m_{\beta}$. Hereafter we will refer to this approximation as the single mass approximation.

In Ref. [6], it has been shown that for energies $E_{\nu}=E_{0}-E \gg m_{i}$, the distortion of the electron energy spectrum due to non-zero neutrino mass and mixing is determined by an effective mass

$$
m_{\beta 1}=\sqrt{\sum_{i} m_{i}^{2}\left|U_{e i}\right|^{2}}
$$

However, the highest sensitivity to the mass of $\nu_{i}$ appears in the energy range close to the end point where $E_{\nu} \sim m_{i}{ }^{\dagger}$ It was shown in Ref. [7] that for $E_{\nu} \sim m_{i}$, the integral spectrum

\footnotetext{
${ }^{\dagger}$ In Ref. [6] it was also noted that Eq. (6) gives a very good fit for $E_{\nu} \sim m_{i}$ too.
} 
can be described by the effective mass

$$
m_{\beta 2}=\sum_{i} m_{i}\left|U_{e i}\right|^{2}
$$

Recently, the approximations based on the effective masses $m_{\beta 1}$ and $m_{\beta 2}$ have been discussed in Ref. [8, 9]. It was claimed in Ref. [8] that for large energy intervals, $\delta \gg m_{i}$, the definition in Eq. (6) provides a better fit of the exact beta decay spectrum, and consequently the mass $m_{\beta 1}$ should be used in future analyses of the experimental results. We do not agree with this last statement. In this paper we consider the issue in more detail and discuss various aspects of the problem. We also expand the analysis for $4 \nu$-schemes.

The paper is organized as follows. In Sect. 2, we compare the two definitions of effective mass for the quasi-degenerate mass schemes. In Sect. 3, we explore the effect of using each form of effective mass on the integrated energy spectrum in quasi-degenerate $3 \nu$-schemes. In Sect. 4 , we perform the same analysis for non-degenerate $3 \nu$-schemes. In Sect. 5 , we address the question of whether it is possible to fit $4 \nu$-schemes by a single parameter, and evaluate the corresponding error. Conclusions are presented in Sect. 6.

\section{Effective masses for quasi-degenerate neutrinos}

The quasi-degenerate mass scheme is the only $3 \nu$-scheme that will cause an observable shift of the end-point in forthcoming $\beta$-decay experiments. We show here that although the combinations in Eqs. (6) and (7) are derived using completely different analyses, they coincide up to corrections of order of $(\Delta m / m)^{2}$ for the quasi-degenerate mass scheme.

We represent the neutrino mass eigenstates as $m_{i}=m_{j}+\Delta m_{i j}$, where $i=1,2,3$ and $m_{j}$ can be any of the mass eigenstates. The expressions for $\left(m_{\beta 1}\right)^{2}$ and $\left(m_{\beta 2}\right)^{2}$ can then be written as

$$
m_{\beta 1}^{2}=m_{j}^{2}+2 m_{j} \sum_{i} \Delta m_{i j}\left|U_{e i}\right|^{2}+\sum_{i}\left(\Delta m_{i j}\right)^{2}\left|U_{e i}\right|^{2}
$$

and

$$
m_{\beta 2}^{2}=m_{j}^{2}+2 m_{j} \sum_{i} \Delta m_{i j}\left|U_{e i}\right|^{2}+\left(\sum_{i} \Delta m_{i j}\left|U_{e i}\right|^{2}\right)^{2} .
$$

These two expressions coincide up to the last terms, which are of order $\left(\Delta m_{i j}\right)^{2}$. Furthermore, the difference turns out to be even smaller if one considers realistic scenarios. According to the experimental data, $\nu_{e}$ is mainly distributed in two states (for definiteness, 
in $\nu_{1}$ and $\left.\nu_{2}\right)$ with mass splitting $\Delta m_{12}^{2} \equiv \Delta m_{\text {sun }}^{2}$ while the contribution of the third state (with splitting $\Delta m_{13}^{2}=\Delta m_{a t m}^{2}$ ) to $\nu_{e}$ is small: $\left|U_{e 3}\right|^{2}<0.04$. Taking $j=1$ we obtain

$$
\frac{m_{\beta 1}^{2}-m_{\beta 2}^{2}}{m_{\beta 2}^{2}} \sim \frac{1}{4 m_{1}^{4}} \operatorname{Max}\left[\left(\Delta m_{\text {sun }}^{2}\right)^{2}\left|U_{e 2}\right|^{2},\left(\Delta m_{\text {atm }}^{2}\right)^{2}\left|U_{e 3}\right|^{2}\right]<10^{-5} .
$$

The numerical bound corresponds to $m_{1}>0.3 \mathrm{eV}$. Clearly, this mass difference is unobservable. This means that $m_{\beta 1}$ and $m_{\beta 2}$ are equally good for the description of the spectrum both near the end point and far from it.

For comparison, we consider another possible definition for the effective mass:

$$
m_{\beta 3}=m_{1}
$$

In this case according to (8), the relative difference of mass squared is linear in $\Delta m_{i j}$ :

$$
\frac{m_{\beta 1}^{2}-m_{\beta 3}^{2}}{m_{\beta 3}^{2}}=2 \sum_{i}\left|U_{e i}\right|^{2} \frac{\Delta m_{i 1}}{m_{1}} \sim \frac{1}{2 m_{1}^{2}} \operatorname{Max}\left[\Delta m_{\text {sun }}^{2}\left|U_{e 2}\right|^{2}, \Delta m_{a t m}^{2}\left|U_{e 3}\right|^{2}\right]<10^{-3},
$$

where the last number corresponds to $\left|U_{e 3}\right|^{2}=0.04$ and $m_{1}>0.3 \mathrm{eV}$. Even in this case the difference between the effective masses is negligible.

\section{Effective masses and the integrated spectrum}

The KATRIN experiment can operate in two modes [10]: i) as an integrating spectrometer (MAC-E-FILTER mode); ii) as a non-integrating spectrometer (MAC-E-TOF mode). In the MAC-E-FILTER mode, the measured quantity is the number of electrons in an interval $\delta$ close to the end-point. For this mode, the energy resolution $(\Delta E \sim 1 \mathrm{eV})$ is a measure of the sharpness of the filter. In the MAC-E-TOF mode, the Time Of Flight of retarded electrons is measured to derive the energy of the electron. The resolution function is triangular with a width of $\sim 1 \mathrm{eV}$.

KATRIN will operate primarily in the integrating mode and we therefore focus here on the integrated energy spectrum. Let us study the error in the integrated energy spectrum caused by using the different definitions for the effective mass. Since $R(E)$ in Eq. (2) is a slowly varying function of energy we can write the number of events above the energy $E_{0}-\delta$ as

$$
n_{a}(\delta)=\bar{R} \int_{E_{0}-\delta}^{E_{0}} \frac{1}{R} \frac{d N_{a}}{d E} d E, \quad(a=0,1,2)
$$

where the subscript $a$ identifies the distribution used; $a=0$ indicates the exact spectrum in Eq. (4), while $a=1,2$ indicate the spectra with effective masses $m_{\beta 1}$ and $m_{\beta 2}$, respectively. 
Here, $E_{0}$ is the end-point of the spectrum for zero neutrino mass and $\bar{R}$ is the averaged value of the function $R(E)$.

The integration in Eq. (12) leads to

$$
n_{0}(\delta)=\frac{\bar{R}}{3} \sum_{i}\left|U_{e i}\right|^{2}\left(\delta^{2}-m_{i}^{2}\right)^{3 / 2}, \quad n_{a}(\delta)=\frac{\bar{R}}{3}\left(\delta^{2}-m_{\beta a}^{2}\right)^{3 / 2} .
$$

Let us define the ratios

$$
r_{a} \equiv \frac{n_{a}-n_{0}}{n_{a}}, \quad a=1,2
$$

which give the errors caused by the single mass approximation (provided that the errors are small). Using Eq. (13) we get

$$
r_{a}=1-\frac{\sum_{j}\left|U_{e j}\right|^{2}\left(\delta^{2}-m_{j}^{2}\right)^{3 / 2}}{\left(\delta^{2}-m_{\beta a}^{2}\right)^{3 / 2}} .
$$

We introduce

$$
\Delta m_{j a} \equiv m_{j}-m_{\beta a}
$$

as we have seen, for quasi-degenerate mass schemes $\Delta m_{j a} \ll m_{\beta a}$. Then, using the smallness of the ratio

$$
\frac{2 m_{\beta a} \Delta m_{j a}}{\delta^{2}-m_{\beta a}^{2}} \ll 1,
$$

we can expand expression Eq. (15) as follows:

$$
r_{a}=\frac{3 m_{\beta a}}{\delta^{2}-m_{\beta a}^{2}} \sum_{j}\left|U_{e j}\right|^{2} \Delta m_{j a}+\frac{3\left(\delta^{2}-2 m_{\beta a}^{2}\right)}{2\left(\delta^{2}-m_{\beta a}^{2}\right)^{2}} \sum_{j}\left|U_{e j}\right|^{2}\left(\Delta m_{j a}\right)^{2}+\mathcal{O}\left(\frac{(\Delta m)^{3}}{\delta^{3}}\right) .
$$

First we consider the effective mass $m_{\beta 2}$, i.e., $a=2$. For $m_{\beta 2}$ the first term in Eq. (18) vanishes. This can be verified very easily; $\sum_{j}\left|U_{e j}\right|^{2}\left(m_{j}-m_{\beta 2}\right)=\sum_{j}\left|U_{e j}\right|^{2} m_{j}-m_{\beta 2}=0$, where we have taken into account the unitarity condition $\sum_{j}\left|U_{e j}\right|^{2}=1$. This cancellation motivated us to introduce the effective mass given by Eq. (7) in Ref. [7]. As a consequence, for $m_{\beta 2}$ the deviation, $r_{2}$ is of $\mathcal{O}\left((\Delta m)^{2}\right)$ :

$$
r_{2}=\frac{3}{2\left(\delta^{2}-m_{\beta 2}^{2}\right)}\left(1-\frac{m_{\beta 2}^{2}}{\delta^{2}-m_{\beta 2}^{2}}\right) \sum_{j}\left|U_{e j}\right|^{2}\left(\Delta m_{j 2}\right)^{2}
$$

For $\delta \sim 1 \mathrm{eV}$, it can be estimated as

$$
r_{2} \sim \sum_{j}\left|U_{e j}\right|^{2} \frac{\left(\Delta m_{j 2}\right)^{2}}{\delta^{2}} \sim \frac{1}{(m \delta)^{2}} \operatorname{Max}\left[\left(\Delta m_{\text {sun }}^{2}\right)^{2}\left|U_{e 2}\right|^{2},\left(\Delta m_{a t m}^{2}\right)^{2}\left|U_{e 3}\right|^{2}\right]<4 \times 10^{-6} .
$$


Notice that the sum in Eq. (19) can be written as

$$
\sum_{j}\left|U_{e j}\right|^{2}\left(\Delta m_{j 2}\right)^{2}=\sum_{j}\left|U_{e j}\right|^{2} m_{j}^{2}-m_{\beta 2}^{2}=m_{\beta 1}^{2}-m_{\beta 2}^{2}
$$

For the effective mass $m_{\beta 1}$, Eq. (18) yields:

$$
r_{1}=-\frac{3 m_{\beta 1}^{2}}{2\left(\delta^{2}-m_{\beta 1}^{2}\right)^{2}} \sum_{j}\left|U_{e j}\right|^{2}\left(\Delta m_{j 1}\right)^{2} .
$$

Again, the sum can be written in terms of effective masses, so that

$$
r_{1}=-\frac{3 m_{\beta 1}^{3}\left(m_{\beta 1}-m_{\beta 2}\right)}{\left(\delta^{2}-m_{\beta 1}^{2}\right)^{2}} .
$$

For $\delta=1 \mathrm{eV}, r_{1}$ and $r_{2}$ have the same orders of magnitude given by Eq. (20).

According to Eqs. $(19,22)$ the ratio of errors is equal to

$$
\left|\frac{r_{2}}{r_{1}}\right|=\frac{\delta^{2}-2 m_{\beta}^{2}}{m_{\beta}^{2}} .
$$

From this we see that

$$
r_{2}<r_{1} \quad \text { for } \quad \delta<\sqrt{3} m_{\beta} \sim \sqrt{3} m_{1}
$$

the approximation with $m_{\beta 2}$ works better than the one with $m_{\beta 1}$ for $\delta$ not too large. For $\delta>\sqrt{3} m_{\beta}$ the spectrum with $m_{\beta 1}$ gives a better fit.

We now consider the error for large $\delta$. Expanding the general formula in Eq. (18) in powers of $1 / \delta^{2}$, we find that for $\delta \gg m_{\beta}$,

$$
r_{a}=\frac{3}{2 \delta^{2}}\left(\sum_{j}\left|U_{e j}\right|^{2} m_{j a}^{2}-m_{\beta a}^{2}\right)+\mathcal{O}\left(\frac{m_{\beta}^{4}}{\delta^{4}}\right) .
$$

For $m_{\beta 1}$, the corrections of order $1 / \delta^{2}$ vanish. The explicit dependences of $r_{a}$ on $\delta$ are given by Eqs. $(19,22)$. For $\delta \rightarrow \infty, r_{1} \propto 1 / \delta^{4}$ and $r_{2} \propto 1 / \delta^{2}$; as $\delta$ increases both approximations converge to the exact result, however, the spectrum with $m_{\beta 1}$ converges faster than the one with $m_{\beta 2}$.

We now use these considerations to discuss the results of [8], where the quantity

$$
h\left(\delta, m_{1}\right)=\frac{\left|n_{0}\left(\delta, m_{1}\right)-n_{2}\left(\delta, m_{1}\right)\right|}{\left|n_{0}\left(\delta, m_{1}\right)-n_{1}\left(\delta, m_{1}\right)\right|}
$$

has been studied for different values of $m_{1}$ (the lightest neutrino mass). For $m_{1}=0.1 \mathrm{eV}$ (a typical quasi-degenerate mass scheme) and small values of $\delta$, the ratio $h\left(\delta, m_{1}\right)$ is smaller 
than one. As $\delta$ increases, $h\left(\delta, m_{1}\right)$ diverges. This is the basis of statement in [8] that the spectrum with $m_{\beta 1}$ gives a better approximation.

According to our results, for large $\delta$,

$$
h=\frac{r_{2}}{r_{1}} \propto \delta^{2},
$$

so that $h$ increases with $\delta$. However, this does not mean that the spectrum with $m_{\beta 2}$ fails to describe the exact integrated spectrum, or that it is worse than the one corresponding to $m_{\beta 1}$. In fact, for the quasi-degenerate mass spectrum in Eq. (27) both the numerator and denominator are very small ( see Eq. (20)), and therefore the ratio $h$ has no real meaning.

For large $\delta$ the difference of the two approximations is unobservable in spite of the possible accumulation of absolute values of deviations. Let us consider this in more detail. According to Eq. (13), the total number of events increases with $\delta$ since $n_{a} \propto \delta^{3}$. On the other hand, the difference of numbers of events increases as

$$
n_{2}-n_{0}=n_{2} \cdot r_{2} \propto \delta
$$

The statistical errors increase faster, $\sqrt{n_{a}} \propto \delta^{3 / 2}$, and therefore, the sensitivity of the experiment to the deviation of the $m_{\beta 2}$-approximation from exact spectrum (4) decreases with the increase of $\delta$. Similarly we find $n_{1}-n_{0} \propto 1 / \delta$, so that even the deviation in absolute number of events decreases for the effective mass $m_{\beta 1}$. Furthermore, if the two approximations are indistinguishable near the end point it is not possible to distinguish them by increasing the integration region. Even in the case that the absolute difference of events increases, the statistical error increases faster.

\section{Non-degenerate $3 \nu$-schemes}

In this section we consider schemes for which the heaviest mass is of order $\sqrt{\Delta m_{\text {atm }}^{2}} \sim$ 0.05-0.07 eV, which is far below the reach of KATRIN experiment. Therefore, the discussion here is relevant only for hypothetical future experiments with substantially better sensitivity and higher energy resolution. We expect that for $\beta$-decay experiments in the near future, $\Delta E, \delta \gg m_{1}, m_{2}$. Consequently, using Eq. (15) we can write

$$
r_{a}=\frac{3}{2} \frac{\sum_{i} m_{i}^{2}\left|U_{e i}\right|^{2}-m_{\beta a}^{2}}{\delta^{2}}-\frac{3}{8} \frac{\sum_{i} m_{i}^{4}\left|U_{e i}\right|^{2}}{\delta^{4}}-\frac{15}{8} \frac{m_{\beta a}^{4}}{\delta^{4}}+\frac{9}{4} \frac{m_{\beta a}^{2} \Sigma_{i} m_{i}^{2}\left|U_{e i}\right|^{2}}{\delta^{4}} .
$$

We first study schemes for which $m_{1} \simeq m_{2} \sim \sqrt{\Delta m_{\text {atm }}^{2}}$ (we remind the reader that the electron-neutrino is mainly distributed in the 1- and 2-states with $\left|m_{1}^{2}-m_{2}^{2}\right|=\Delta m_{\text {sun }}^{2}$.) 
Note that this includes the inverted hierarchical scheme as well as the normal scheme with a non-zero lightest mass. If in Eq. (30) we set $m_{\beta a}$ equal to either $m_{\beta 2}, m_{1}$ or $m_{2}$, the error will be of order

$$
\operatorname{Max}\left[\left(\frac{\Delta m_{a t m}^{2}}{\delta^{2}}\right)\left|U_{e 3}\right|^{2},\left(\frac{\Delta m_{a t m}^{2}}{\delta^{2}}\right)^{2},\left(\frac{\Delta m_{\text {sun }}^{2}}{\delta^{2}}\right)\right],
$$

which will probably be smaller than the experimental errors; it is safe to use $m_{\beta 2}$. However, $m_{\beta 1}$ gives an even better fit:

$$
r_{1} \sim \operatorname{Max}\left[\frac{\left(\Delta m_{\text {sun }}^{2}\right)^{2}}{\delta^{4}},\left(\frac{\Delta m_{\text {atm }}^{2}}{\delta^{2}}\right)^{2}\left|U_{e 3}\right|^{2}\right],
$$

where we have assumed that $m_{3}$ is also of the order of $\sqrt{m_{a t m}^{2}}$.

We now discuss the normal hierarchical mass scheme, $m_{1} \ll m_{2} \simeq \sqrt{\Delta m_{\text {sun }}^{2}} \ll m_{3} \simeq$ $\sqrt{\Delta m_{\text {atm }}^{2}}$, neglecting the effect of the lightest state. If $\left|U_{e 3}\right|$ is close to its present upper bound, we can also neglect the second state, so that

$$
m_{\beta 1} \approx m_{3}\left|U_{e 3}\right|, \quad m_{\beta 2} \approx m_{3}\left|U_{e 3}\right|^{2}
$$

In this case $\left(m_{\beta 1}-m_{\beta 2}\right) / m_{\beta 1} \sim 1$, and in fact, $m_{\beta 1} \gg m_{\beta 2}$. The effect of the neutrino mass would consist of a kink at $E=E_{0}-m_{3}$ whose size is determined by $\left|U_{e 3}\right|^{2}$. For the realistic case $\delta \gg m_{3}$, the statistics should be very high to detect the deficit of the total number of events above $E_{0}-\delta$. In this case we can use a single effective mass instead of several parameters; the error $r_{a}$ defined in Eq. (14), is negligible for an appropriate choice of effective mass. For $m_{\beta 1}$, the first term in Eq. (30) vanishes, and the $m_{\beta 1}$ approximation gives a very good description of the data. In contrast, $m_{\beta 2}$ does not describe the situation well; the deviation of the $m_{\beta 2}$ approximation from the exact spectrum is comparable to the effect of non-zero mass itself.

\section{Four-neutrino mass schemes}

In this section we study the effective mass approximation for the case of $4 \nu$-schemes motivated by the results of the LSND experiment [13]. (Apart from the LSND results, there are other motivations for the existence of a 4th neutrino. One can use the arguments described below for more general cases of active-sterile neutrino mixing.)

The main feature of these schemes is the existence of two groups of states separated by a large mass gap given by $\Delta m_{L S N D}^{2} \sim 1 \mathrm{eV}^{2}$. The mass splitting within each group is small: 
$\Delta m^{2} \ll \Delta m_{L S N D}^{2}{ }^{\ddagger}$ Furthermore, the admixture of $\nu_{e}$ in one of these groups is very small; it is restricted by the reactor experiments CHOOZ and BUGEY.

In $3+1$ schemes $\nu_{e}$ is mainly distributed in three mass eigenstates with small mass differences given by $\Delta m_{\text {sun }}^{2}$ and $\Delta m_{\text {atm }}^{2}$, while the fourth eigenstate (separated by a mass gap $\Delta m_{L S N D}^{2}$ from the rest of the states) has a small admixture of the electron neutrino, i.e., $\left|U_{e 4}\right| \ll 1$.

In $2+2$ scheme, $\nu_{e}$ is mainly distributed in the first and second mass eigenstates with a small splitting given by $\Delta m_{\text {sun }}^{2}$. The third and fourth mass eigenstates, separated from the rest of the states by $\Delta m_{L S N D}^{2}$, have small admixtures of $\nu_{e}$ given by $\left|U_{e 3}\right|^{2}$ and $\left|U_{e 4}\right|^{2}$.

We identify the groups of mass eigenstates as $h$ - (heavy) and $l$ - (light) groups. As discussed in Ref. [7], the effect of each group on the beta decay spectrum can be described by only two parameters:

$$
\rho_{a}=\Sigma_{i}\left|U_{e i}\right|^{2} \quad \text { and } \quad m_{a}=\Sigma_{i} m_{i}\left|U_{e i}\right|^{2} / \rho_{a}, \quad(a=h, l),
$$

where $i$ runs over the members of each group and $m_{h}^{2}-m_{l}^{2} \simeq \Delta m_{L S N D}^{2}$. Unitarity implies $\rho_{l}+\rho_{h}=1$. The effect of the heavier group is a kink whose size and position are given by $\rho_{h}$ and $m_{h}$, respectively. The lighter group leads to a shift of the end-point. As for the case of the $3 \nu$-scheme it can be shown that the error due to using the effective mass parameters $m_{a}$ is negligible.

We denote

$$
\rho_{e} \equiv \operatorname{Min}\left[\rho_{h}, \rho_{l}\right]
$$

in the $(3+1)$ scheme $\rho_{e}=\left|U_{e 4}\right|^{2}$, while in the $(2+2)$ scheme $\rho_{e}=\left|U_{e 3}\right|^{2}+\left|U_{e 4}\right|^{2}$. In the range of $\Delta m_{L S N D}^{2} \sim(0.3-2) \mathrm{eV}^{2}$, the strongest upper bound on $\rho_{e}$ follows from the BUGEY experiment [11]:

$$
\rho_{e}<0.027(90 \%) \quad \text { C.L. }
$$

In what follows we will refer to the scheme in which the heavy set has a small admixture of $\nu_{e}, \rho_{h}=\rho_{e}$, as "normal" scheme. The scheme in which the light group has the smaller contribution to $\nu_{e}, \rho_{l}=\rho_{e}$, will be called the "inverted" scheme.

For the normal schemes (either $2+2$ or $3+1$ ), the beta spectrum should have a "small" kink at $E_{0}-m_{h}$, with a height characterized by $\rho_{e}$ and the end point will be shifted to $E_{0}-m_{l}$. In contrast, for the inverted schemes we expect a "large" kink of size $1-\rho_{e} \approx 1$

\footnotetext{
${ }_{\ddagger}^{\ddagger}$ Clearly, there is no splitting if the group consists only of one state.
} 
at $E_{0}-m_{h}$, and a small "tail" after the kink with a height $\propto \rho_{e}$. In general, the spectrum with a given type of hierarchy is described by three parameters: $\rho_{e}, m_{h}$, and $m_{l}$.

Suppose a priori we do not know about the existence of the 4th neutrino, and therefore try to fit the integrated beta spectrum using the single mass approximation:

$$
n_{s}(\delta)=\frac{\bar{R}}{3}\left(\delta^{2}-m_{f}^{2}\right)^{3 / 2}
$$

where $m_{f}$ is the fit mass parameter. We clarify below the meaning of such a fit.

1) We consider first the normal mass scheme. Due to the smallness of $\rho_{e}$, the main effect will be produced by $m_{l}$. The single mass fit (Eq. (35)) means that we neglect the kink and set $m_{f} \simeq m_{l}$. Let us estimate the error due to neglecting the kink, $\left(m_{f}-m_{l}\right) / m_{l}$.

If $\delta<m_{h}$, the energy interval does not contain the kink, and we can write

$$
n(\delta)=\frac{\bar{R}}{3}\left(1-\rho_{e}\right)\left(\delta^{2}-m_{l}^{2}\right)^{3 / 2} .
$$

Then, fitting $n(\delta)$ with $n_{s}(\delta)$ (that is, equating $\left.n(\delta)=n_{s}(\delta)\right)$ we find

$$
\frac{m_{f}-m_{l}}{m_{l}} \approx \frac{\rho_{e}}{3} \frac{\left(\delta^{2}-m_{l}^{2}\right)}{m_{l}^{2}},
$$

where we have used the smallness of $\rho_{e}$. According to this expression, the mass $m_{f}$ which we obtain from the single mass fit is larger than the exact mass $m_{l}$. The relative mass difference increases with $\delta$ and decreases with $m_{l}$. For $m_{l}=0.3 \mathrm{eV}$ and $\delta=1 \mathrm{eV}$, using the bound in Eq. (34), we obtain $\left(m_{f}-m_{l}\right) / m_{l}<0.1$. That is the error is smaller than $10 \%$.

If $\delta>m_{h}$ (i.e., the energy interval contains the kink), the number of events amounts to

$$
n(\delta)=\frac{\bar{R}}{3}\left[\rho_{e}\left(\delta^{2}-m_{h}^{2}\right)^{3 / 2}+\left(1-\rho_{e}\right)\left(\delta^{2}-m_{l}^{2}\right)^{3 / 2}\right] .
$$

Fitting $n(\delta)$ with the approximate spectrum $n_{s}(\delta)$ in Eq. (35), we obtain

$$
\frac{m_{f}-m_{l}}{m_{l}}=\frac{\rho_{e}}{3} \frac{\left(\delta^{2}-m_{l}^{2}\right)^{3 / 2}-\left(\delta^{2}-m_{h}^{2}\right)^{3 / 2}}{\left(\delta^{2}-m_{l}^{2}\right)^{1 / 2} m_{l}^{2}},
$$

where we have neglected the higher orders of $\rho_{e}$. The relative difference increases with $\delta$ and in the limit $\delta \gg m_{h}$ asymptotically approaches to

$$
\frac{m_{f}-m_{l}}{m_{l}}=\frac{\rho_{e}}{2} \frac{\Delta m_{L S N D}^{2}}{m_{l}^{2}} .
$$

For $\Delta m_{L S N D}^{2}=1 \mathrm{eV}^{2}$ and $m_{l}=0.3 \mathrm{eV}$ using Eq. (34), we find that $\left(m_{f}-m_{l}\right) / m_{l}<15 \%$. This can also be interpreted in this way: the effect of the small kink can be resolved only if the experimental errors are smaller than $\sim 15 \%$. 
2) We now consider the inverted schemes. In these cases the main effect is a large kink at $E_{0}-m_{h}$. Fitting the spectrum with a single mass, we are not sensitive to the small tail after the kink and the fit parameter $m_{f}$ corresponds to $m_{h}$. Let us evaluate the relative difference of $m_{f}$ and $m_{h}$. The exact spectrum is

$$
n(\delta)=\frac{\bar{R}}{3}\left[\rho_{e}\left(\delta^{2}-m_{l}^{2}\right)^{3 / 2}+\left(1-\rho_{e}\right)\left(\delta^{2}-m_{h}^{2}\right)^{3 / 2}\right] .
$$

(It corresponds to $n(\delta)$ for the normal scheme substituting $h \leftrightarrow l$.) Fitting $n(\delta)$ with $n_{s}(\delta)$, we obtain:

$$
\frac{m_{h}-m_{f}}{m_{h}}=\frac{\rho_{e}}{3} \frac{\left(\delta^{2}-m_{l}^{2}\right)^{3 / 2}-\left(\delta^{2}-m_{h}^{2}\right)^{3 / 2}}{\left(\delta^{2}-m_{h}^{2}\right)^{1 / 2} m_{h}^{2}} .
$$

Notice that for the inverted schemes $m_{h}>m_{f}$. The absolute value of the relative difference, as in the case of the normal scheme, is limited by the quantity in the right-hand side of Eq. (40).

Most probably, KATRIN will start operation, after results of the MiniBooNE experiment [12] are released. If MiniBooNE does not see any oscillation effect, a stronger bound on $\rho_{e}$ will be obtained and any possible effect of a fourth neutrino on the beta spectrum will be further suppressed. If the MiniBoone experiment confirms the LSND results, the existence of a 4 th neutrino will be confirmed and the values of $\Delta m_{L S N D}^{2}$ and $\rho_{e}$ will be determined. However, MiniBooNe will not be able to discriminate between the normal and inverted schemes so, a priori we do not know which formula, Eq. (38) or Eq. (41), describes the exact spectrum.

KATRIN not only can determine the neutrino mass scale, but also can help to discriminate between normal and inverted schemes. In this direction, the following strategy can be used:

First, a fit of the data using single mass approximation given by (35) can be performed. Then the value of the fit parameter $m_{f}^{2}$ should be compared to $\Delta m_{L S N D}^{2}$. If $m_{f}^{2}<\Delta m_{L S N D}^{2}$, considering the fact that $m_{h} \geq \sqrt{\Delta m_{L S N D}^{2}}$ we can conclude that the scheme is normal and that $m_{f} \approx m_{l}$. To extract the value of $m_{l}$ more accurately (if the experimental uncertainties allow), we can invoke Eq. (38). If $m_{f}^{2} \geq \Delta m_{L S N D}^{2}$, the situation is ambiguous. In this case, either of the following schemes is possible:

a) an inverted mass scheme with $m_{h}^{2}=m_{f}^{2}$ and $m_{l}^{2}=m_{f}^{2}-\Delta m_{L S N D}^{2}$, or

b) a normal mass hierarchy with $m_{l}^{2}=m_{f}^{2}$ and $m_{h}^{2}=m_{f}^{2}+\Delta m_{L S N D}^{2}$.

In principle, this ambiguity can be solved by a non-integrating spectrometer. If a small kink around $E_{0}-\sqrt{m_{f}^{2}+\Delta m_{L S N D}^{2}}$ is observed, the scheme is normal.

Note that for $m_{f}^{2}>\Delta m_{L S N D}^{2}$, the error $\left(\left(m_{l}-m_{f}\right) / m_{f}\right.$ for the normal schemes and $\left(m_{h}-\right.$ 
$\left.m_{f}\right) / m_{f}$ for the inverted schemes) is less than $0.1 \%$, which is negligible. If the accuracy of a hypothetical integrating spectrometer is better than this, by studying the dependence of $m_{f}$ on $\delta$ we can determine whether the scheme is normal or inverted.

Recently to reconcile the LSND results with the results of the other neutrino experiments another scenario has been suggested [14]. In this scenario, there is no need for sterile neutrinos but instead, the masses of neutrinos and anti-neutrinos are different. It is supposed that this phenomenological model originates from a more fundamental non-local theory in which CPT is violated in the neutrino sector [15]. Note that the Tritium beta-decay experiments can only provide information about the masses of anti-neutrinos because in these experiments only the electron anti-neutrino is involved.

In Ref. [16] a model is proposed which can accommodate the LSND results as well as the results of KamLAND and other neutrino experiments. In this model the anti-neutrino sector is composed of three mass eigenstates with splittings $\Delta m_{\text {Kam }}^{2} \sim 10^{-4} \mathrm{eV}^{2}$ and $\Delta m_{L S N D}^{2} \sim$ $1 \mathrm{eV}^{2}$. The electron anti-neutrino is mainly distributed in the states with the small splitting $\left(\Delta m_{K a m}^{2}\right)$ while the contribution of the third state to $\bar{\nu}_{e}$ is bounded by Bugey $\left(\left|U_{e 3}\right|^{2}<0.027\right)$. Since KATRIN will not be able to resolve the small splittings $\Delta m_{K a m}^{2}, \Delta m_{L M A}^{2}$ and $\Delta m_{a t m}^{2}$, the signature of the CPT-violating model described in Ref [16] will be exactly the same as the four-neutrino schemes and any result we have found above applies to this model as well.

\section{Conclusions}

We have discussed the application of the effective mass approximation to the energy spectrum of beta decay for different mass schemes, and have found the following results.

1) For a quasi-degenerate $3 \nu$-scheme, which is the only scheme that can have an observable effect on KATRIN, both definitions of the effective mass, $m_{\beta 1}(6)$ and $m_{\beta 2}(7)$, give a very good fit and are practically indistinguishable. In the analysis of the KATRIN data one can safely use a single effective mass, which can be identified with any of $m_{\beta 1}, m_{\beta 2}$ or $m_{\beta 3}$. The accuracy of the forthcoming measurements will not be enough to distinguish between the different mass definitions. We have found that increasing the integration region decreases the sensitivity to the mass. Notice that if in the future, measurements close to the end point $\left(\delta<2 m_{1}\right)$ with very high sensitivity become possible, $m_{\beta 2}$ will provide a better fit.

2) We have discussed the effect of non-degenerate $3 \nu$-schemes on measurements of hypothetical new experiments sensitive to neutrino mass down to $\sim \sqrt{\Delta m_{\text {atm }}^{2}}$. For an inverted hierarchical $3 \nu$-scheme, the discussions and conclusions are similar to the quasi-degenerate 
case. In the case of a normal mass hierarchy, $m_{\beta 1}$ provides a good fit for the integrated energy spectrum with $\delta=E-E_{0} \gg m_{\beta 1}$. However, the $m_{\beta 2}$ approximation fails in this case, in the sense that the deviation is comparable to the mass effect itself.

3) For the $4 \nu$-schemes motivated by the LSND results, non-zero masses will lead to both a kink in the spectrum shape and a shift of the end-point in beta spectrum [7]. We show that, due to smallness of $\rho_{e}$ (the admixture of the electron neutrino in one of the groups of mass eigenstate) the data can be fitted by a spectrum with a single mass $m_{f}$ to a good approximation. For $m_{f}>0.3 \mathrm{eV}$ (that is, in the range of the KATRIN sensitivity) this mass equals to the exact mass to better than $(10-15) \%$ accuracy.

In summary, in this letter we have clarified the question that which kind of theoretical spectrum should be used to fit the experimental data on beta decay. We have shown that the present knowledge on the oscillation parameters gives rather definite answer to this question which depends on the sensitivity of the experiment to neutrino mass and on the number of neutrino mass eigenstates involved. For $3 \nu$-schemes we have shown that to analyze the results of experiments with sensitivity around that of KATRIN, one can fit the spectrum with a single mass $m_{\beta}$ identified by any of the combinations $m_{\beta i}, i=1,2,3$. In the case of four (or more) neutrino eigenstates, it is also possible to use the single mass approximation. In this case $m_{\beta}$ gives the effective mass of the heavy or light group (depending on the type of the mass scheme) of the quasi-degenerate states with an accuracy better than $20 \%$.

\section{Acknowledgments}

We would like to thank F. Vissani for helpful comments. We are also grateful to F. Petriello and H. Quinn for fruitful comments and careful reading of the manuscript.

\section{References}

[1] F. Boehm and P. Vogel, "Physics of Massive Neutrinos", Cambridge University Press, 1992.

[2] J. Bonn et al., Nucl. Phys. Proc. Suppl. 91 (2001) 273. 
[3] V. M. Lobashev et al., Proc. of the Int. Conf. Neutrino 2000, Sudbury, Canada, Nucl. Proc. Suppl. 77 (1999) 327; V. M. Lobashev et al., Nucl. Phys. Proc. Suppl. 91 (2001) 280; V. M. Lobashev, Prog. Part. Nucl. Phys. 48 (2002) 123.

[4] http://ik1au1.fzk.de/ katrin/

[5] R. E. Shrock, Phys. Lett. B96 (1980) 159.

[6] F. Vissani, hep-ph/0102235 and F. Vissani, Nucl. Phys. Proc. Suppl. 100 (2001) 273.

[7] Y. Farzan, O. L. G. Peres and A. Yu. Smirnov, Nucl. Phys. B612 (2001) 59.

[8] J. Studnik and M. Zralek, hep-ph/0110232.

[9] F. Feruglio, A. Strumia and F. Vissani, Nucl. Phys. B637 (2002) 345.

[10] KATRIN Collaboration (A. Osipowicz et al.), hep-ex/0109033.

[11] Bugey Collaboration, B. Achkar et. al, Nucl. Phys. B434 (1995) 503.

[12] http://www-boone.fnal.gov/

[13] W. Grimus and T. Schwetz, Eur. Phys. J. C20 (2001) 1; M. Maltoni, T. Schwetz and J. W. F. Valle, Phys. Rev. D65 (2002) 093004; M. Maltoni, T. Schwetz, M. A. Tortola and J. W. F. Valle, Nucl. Phys. B643 (2002) 321; hep-ph/0207227; H. Pas, L. Song and T. J. Weiler, hep-ph/0209373.

[14] H. Murayama and T. Yanagida, Phys. Lett. B520 (2001) 263; G. Barenboim, et. al., JHEP 0210 (2002) 001; G. Barenboim, et. al., Phys. lett. B534 (2002) 106.

[15] G. Barenboim and J. Lykken, hep-ph/0210411.

[16] G. Barenboim, et. al.,, hep-ph/0212116. 